\title{
DE-striping hype spectral Remote Sensing Images using Deep Convolutional Neural Network
}

\author{
Atheel Sabih Shaker ${ }^{1}$, Alaa Wagih Abdulqader ${ }^{2}$ and Maad M. Mijwil ${ }^{3}$ \\ ${ }^{1}$ Computer Techniques Engineering Department, Baghdad College of Economic Sciences University, \\ Baghdad, Iraq \\ Email: atheel.sabih [AT] baghdadcollege.edu.iq \\ ${ }^{2}$ Computer Techniques Engineering Department, Baghdad College of Economic Sciences University, \\ Baghdad, Iraq \\ EMail: alaa_wagih [AT] baghdadcollege.edu.iq \\ ${ }^{3}$ Computer Techniques Engineering Department, Baghdad College of Economic Sciences University, \\ Baghdad, Iraq \\ Email: mr.maad.alnaimiy [AT] baghdadcollege.edu.iq
}

\begin{abstract}
Hyperspectral far off detecting records reflectance or emittance information in a huge amount of bordering and tight unearthly groups, and accordingly has numerous data in distinguishing and planning the mineral zones. Then again, the science and natural information gives us some other productive data about the actual qualities of pictures and channels that have been recorded from the surface. In this work, we focus on de-striping the hyperspectral remote sensing images on Hyperion data by applying Deep Convolutional Neural Network (DCNN). What is clear is the high significance of applying the sufficient pre-preparing on Hyperion information as a result of low sign to-commotion proportion. By contrasting the known layers of DCNN model for de-striping hyperspectral pictures. The results obtained by applying the mentioned methods, it is revealed that all the higher stripes in an image as well as black color has been reduced and entirely associated with the Hyperion data alteration, and in contrast, the Hyperion imagery successfully corresponds to the de-striping of hyperspectral image with an accuracy of $91.89 \%$ using DCNN model. The proposed DCNN is capable of reaching high accuracy 150s after the start of the evaluation phase and never reaches low accuracy. The pre-trained DCNN model approach would be an adequate solution considering de-striping as its high inference time is lower compared existing available methods which are not as efficient for de-striping.
\end{abstract}

Keywords— Neural Network, Image, Hyperspectral imagery, Biological data, De-striping, DCNN, Hyperion data.

\section{INTRODUCTION}

From the remote sensing perspective, this research introduces an algorithm for the processing of Hyperion data including all necessary de-striping steps in logical order. The literature, in most of the previous studies, the results are not satisfactory mainly due to the noisy nature of the Hyperion data. Therefore, the main contribution of this study is expected to introduce a comprehensive application that aims to increase the accuracy of the de-striping images and removing all the black from an image as much as possible.

After the correction, the inversion of the calibrated image data into different classes or deep learning proportions will applied. First of all the images of interest need to be identified through a process called neural network endmember identification. There are two general inversion types that may be utilized: hyperspectral image de-striping or hyperspectral image unmixing for striping. While de-striping methods assign a single, unique image endmember to each pixel in an hyperspectral image, unmixing methods are more sophisticated and assign a proportional amount of each hyperspectral image endmember to each pixel. To speed up calculations, focus the problem and simplify the analysis, a subset of the image feature space is often. This is called feature selection process and involves selecting a subgroup of image bands or re-projection and simplification of the feature space for de-striping using deep learning [1].

The hyperspectral image vector space for de-striping over all of the hyperspectral image bands is called feature space. Feature selection and feature extraction are methods for selecting or extracting a subset black color of the feature space for processing. Imaging spectrometer data are, in general, over determined with high band-to-band correlation. The feature selection problem may be approached in a couple of ways. If the hyperspectral sensor has some reported defects on certain bands it is wise to eliminate those bands using DCNN. The simplest approach is to apply knowledge of what 
parts of the spectra are important for a specific problem and use only those selected regions, like for hyperspectral image absorption features are in the DCNN part of the spectrum. The more general approach involves orthogonalization of the hyperspectral image vector space and the use of sub-space projections for de-striping. While technically elegant, orthogonalization can propagate noise and stripes through the hyperspectral image vector space. While it may be used for noise reduction, this is only feasible in the case of a hyperspectral image de-striping model.

\subsection{Research Problem}

We present the primary and secondary questions that this assignment aims to answer.

- Is it possible to de-stripe images by using a Deep Convolutional Neural Network (DCNN) and a measured ground truth of conversion percentage?

- Does the approach of deep learning techniques as a way of automating de-striping process with hyperspectral remote sensing images?

- Do hyperspectral remote sensing images are necessary to obtain good results?

- What ought to be an execution pipeline for productively measure the hyperspectral distant detecting pictures for destriping utilizing DCNN order?

- What angles should be thought of if further work regarding the matter is to be performed?

\subsection{Aim of Study}

This assignment aspires to propose a fully functional de-striping method composed of the following elements:

- Provide an overview of previous works and achievements on de-striping on images.

- Apply DCNN methods hyperspectral remote sensing images to the unified database of striping scans to perform destriping of images which potentially contain stripes.

- Evaluate and compare used DCNN, as well as compare with other studies from the literature.

- Develop a novel methodology with good results for de-striping.

\section{LITERATURE REVIEW}

In article [2] lead research on de-striping measure utilizing inclination greatness histograms with picture surface and information investigation in subset a MR picture. The learning approach utilized solo k-implies bunching learning and accomplished an affectability and explicitness of $87.98 \%$ separately. Author in article [3] show that picture surface investigation and de-striping of pictures in various outputs utilizing information mining with AI and dark level angle coevents are important procedures that accomplish great outcomes. They think about a few AI characterization strategies including Neural Networks, Support Vector Machines and k-Nearest Neighbor that all perform well. Authors in [4] use image features based on ANN for de-striping an image and gray level histograms to de-stripe image regions. The premium features are discovered utilizing a hereditary calculation propelled by the standard of natural development. The upgraded highlight vector is taken care of to a neural organization and they accomplish a de-striping pixel characterization precision of $79.6 \%$. Authors in [5] and [6], seen de-striping and striping independently in 2 investigations. The two investigations included multi-ghostly pictures FFT prepared utilizing highlights chosen utilizing forward stepwise choice. At the point when contrasted utilizing various strategies with various scoring approaches the FFT models were demonstrated to be unrivaled. The best of these models was found to outflank score-based drew nearer by up to $69 \%$ with an outright transformation of $83.45 \%$.

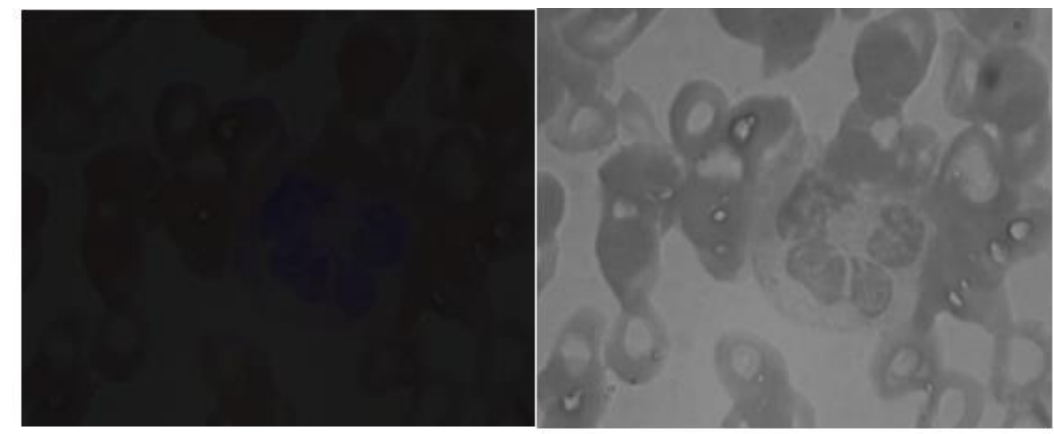

Figure 1: De-striping kernel black from augmented blood cells images using DCNN [7]. 
Many new model architectures emerge as deep learning grows and evolves. As a result, it's critical to assess which of them delivers the greatest results in terms of the amount of produced false positives and time consumption with low rank representation [8]. When it comes to certain tasks, highly deep neural networks can be overkill, While using less sophisticated methods can produce similar results while saving resources [9] using more complex methods can produce similar results while saving resources. As a result, each specific problem necessitates comprehensive and exhaustive research that compare various techniques and provide a report on their efficacy.

Separate models that learn to de-stripe between stripes and non-stripes are used to improve the accuracy of a whole end-to-end de-striping system. The received probability for each striping procedure is then utilized to filter away any stripes that do not contain any black kernel, resulting in a decreased number of images for Hyperion data [10]. Destriping has been accomplished using a variety of methods. Some methods use traditional computer vision techniques to extract features, which are then processed by a machine learning classifier (decision trees, k-Nearest Neighbor, Support Vector Machine, artificial neural network, etc.) [11].

\section{METHODOLOGY}

Deep Convolution Neural Network (DCNN) is a class of neural work that becomes hot lately in light of the tremendous achievement in heaps of undertaking opposite arrangement, picture division, object following has been utilized exclusively for the demonstrating of this exploration work. DCNN was propelled by the availability example of the neurons in the cerebrum. As the name shows, the fundamental bit in DCNN is profound convolution activity. The interaction portrayed as profound convolution here is simply by show in profound learning. Numerically, it is a crossconnection. In PC vision application, convolution is constantly done between bit (channel) and the picture. The principal reason for these activities is separating the highlights. The pre-preparing needed in DCNN is many less than other customary strategies. With enough preparing, DCNN can become familiar with those channels that were hand-designed in conventional techniques for de-striping on hyperspectral distant detecting pictures. This principal benefit of DCNN is that there is no compelling reason to remove the network and plan the equation to separate the highlights physically. The learnable loads and inclination DCNN are a more impressive choices than human exertion and information in include plan.

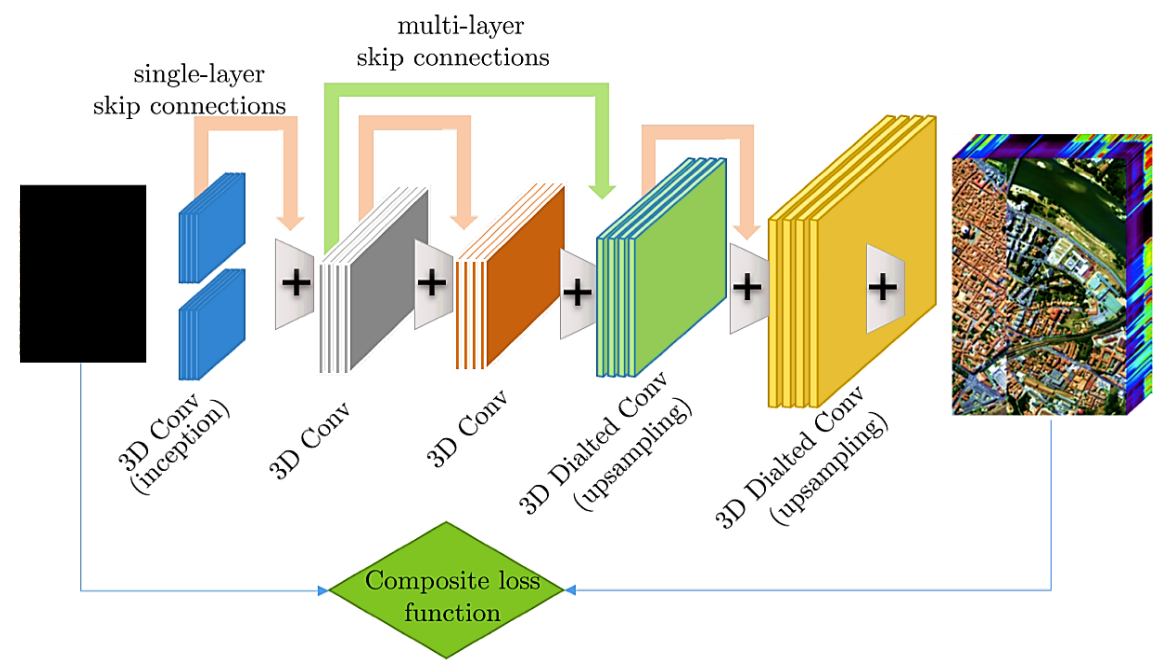

Figure 2: Each layer contains a set of functions that can be plugged together to convolute and finally leading to the destriping.

To be honest, the reason for the task is to give a model or equation that can be effortlessly embedded and adjusted to destripe hyperspectral pictures. Rather than giving recipe, the model adjusted to Hyperion information, a model method is given utilizing DCNN that everybody can continue to de-stripe Hyperion information and pictures in the outcome. The presentation is very encouraging inside the Hyperion information that comes from a similar source. 


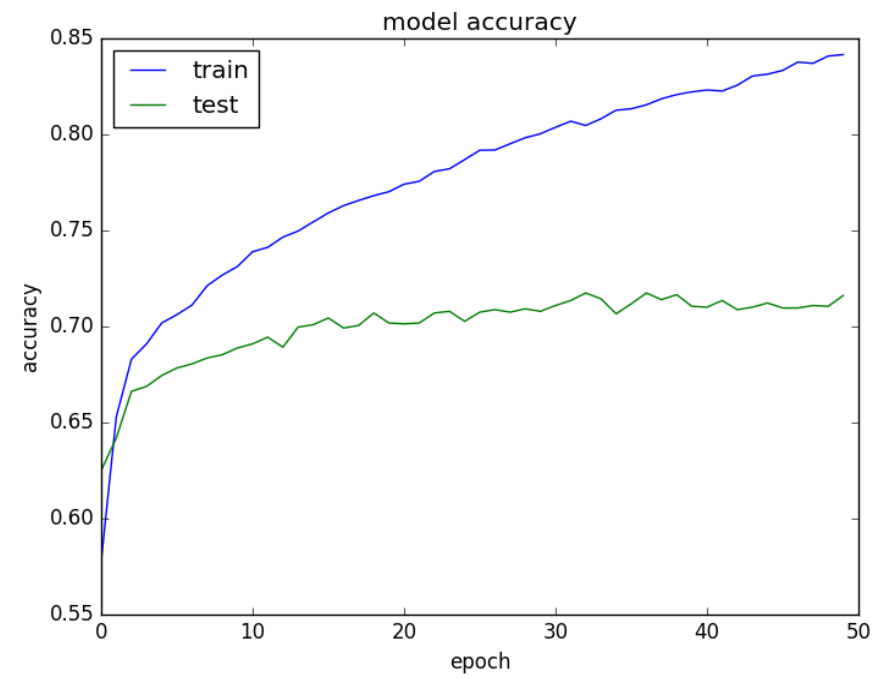

Figure 3: The DCNN model accuracy for training and testing.

As expected, the DCNN model allows for an interesting increase in performance over other baseline models. However, the training time required to fit a model is more consequent for the DCNN than other available existing models for destriping. This enhancement of performance among the de-striping is particularly visible for the DCNN models. The DCNN, on the other hand, is capable of handling most subjects with smaller variations in performance. We distribute the Hyperion data into $85 \%$ for testing and $15 \%$ for the purpose of testing. Regarding the misclassified trials, a quick hyperspectral image analysis of the matrices reveals that DCNN tends to exactly de-stripe. This robustness can be an advantage of Hyperion data partially distributed for de-striping images. The DCNN approach don not seem to suffer from any issue. Training the DCNN took 179.85 seconds and 26.78 seconds for testing each hyperspectral remote sensing image for a nice performance while reducing the average training time.

\section{RESULTS}

This assignment is deemed a necessity in order to achieve a fully automatic method for de-striping of hyperspectral remote sensing images. Below are results of de-striping on some images with improved DCNN methods discussed in this assignment. Hyperion data is available foe the level of correction, thus tests using Hyperion data have been performed to achieve de-striping of images as given below:

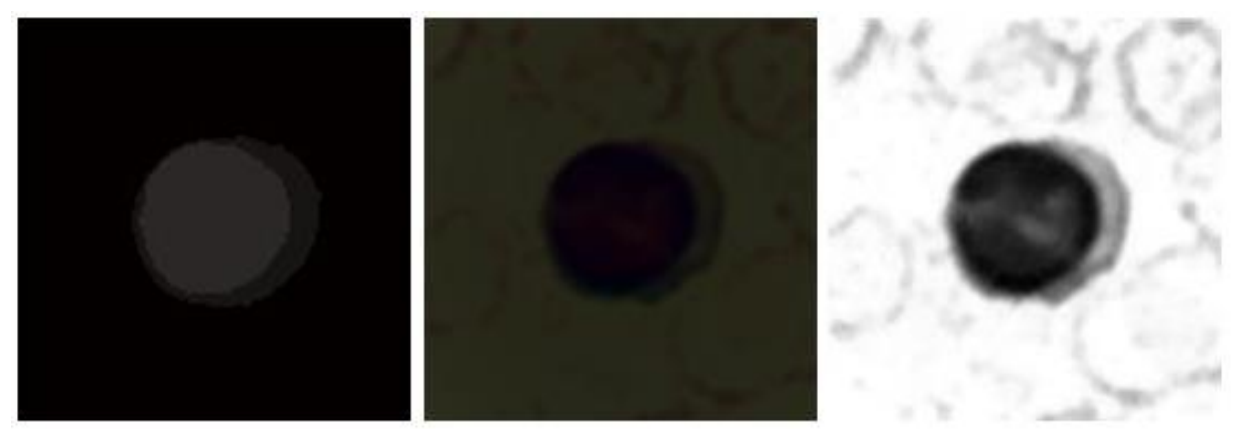

Figure 4: (Step 1) De-striping of an image sample using DCNN.
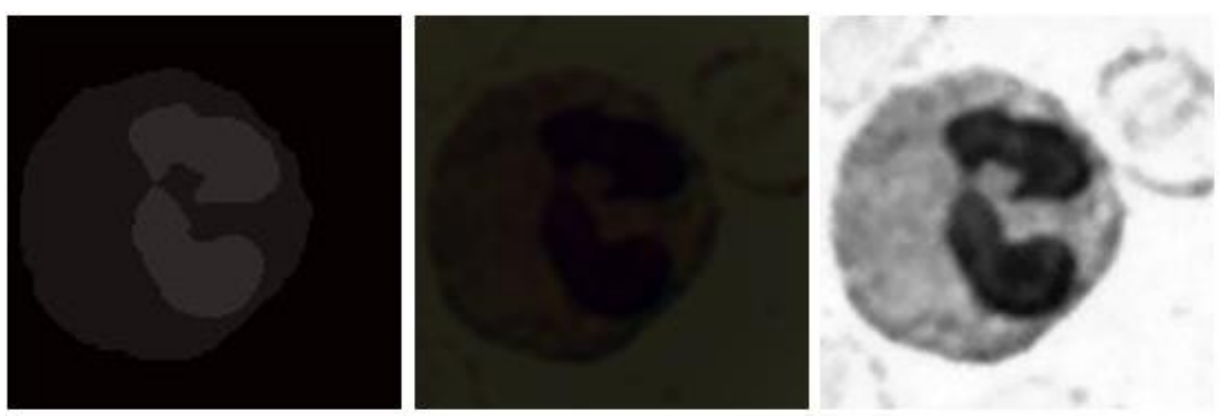

Figure 5: (Step 2) De-striping of an image sample using DCNN. 

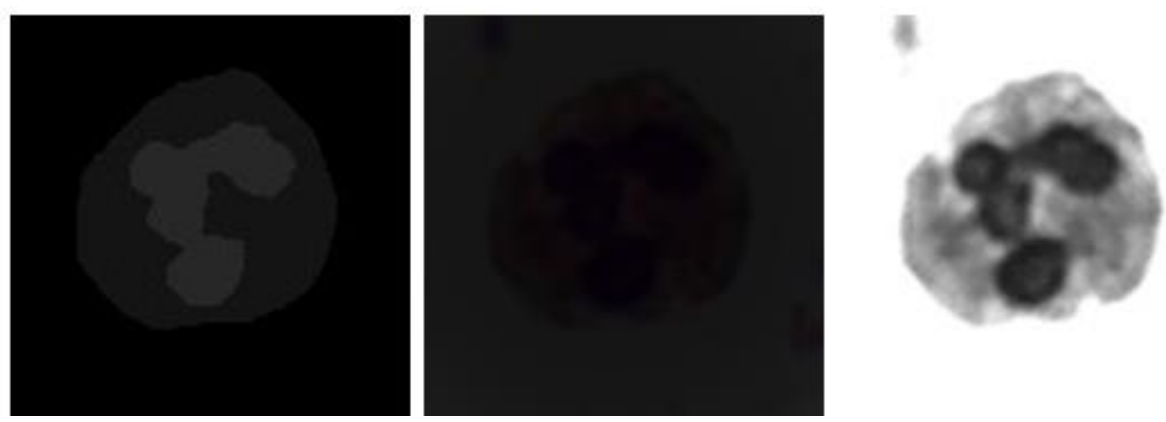

Figure 6: (Step 3) De-striping of an image sample using DCNN.
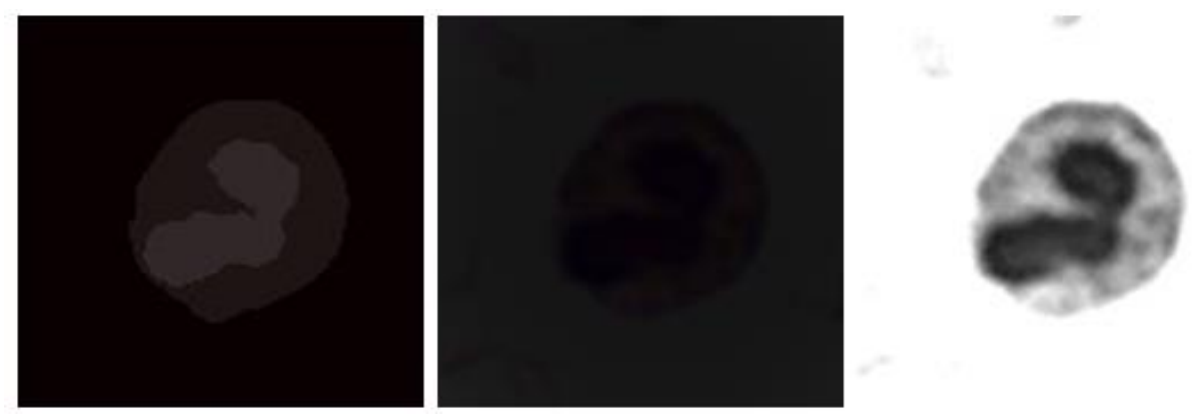

Figure 7: (Step 4) De-striping of an image sample using DCNN.

\section{CONCLUSION}

We provided an overview of previous works and achievements on de-striping on images. We applied DCNN methods on hyperspectral remote sensing images to the unified database of striping scans to perform de-striping of an image which potentially contain stripes. Pre-trained model for each input trial from which we extract detripe hyperspectral image data with an accuracy of $91.89 \%$. The processing operations consist of different layers of DCNN. All other parameters remain identical to the de-striping of Hyperion data-based image. The proposed DCNN is capable of reaching high accuracy 150s after the start of the evaluation phase and never reaches low accuracy. The pre-trained DCNN model approach would be an adequate solution considering de-striping as its high inference time is lower compared existing available methods which are not as efficient for de-striping.

\section{REFERENCES}

[1] Mijwil, M. M., and Abttan R. A., “Artificial Intelligence: A Survey on Evolution and Future Trends,” Asian Journal of Applied Sciences (AJAS), vol.9, no.2, pp:87-93, 29 April 2021, Pakistan, https://doi.org/10.24203/ajas.v9i2.6589

[2] B. Munch, P. Trtik, F. Marone, and M. Stampanoni, "Stripe and ring artifact removal with combined wavelet Fourier filtering and MR," Opt. Express., vol. 17, no. 10, pp. 8567-8591, May 2017.

[3] R. Pande-Chhetri and A. Abd-Elrahman, "De-striping hyperspectral imagery using wavelet transform and adaptive frequency domain filtering using machine learning," ISPRS J. Photogramm. Remote Sens., vol. 66, no. 5, pp. 620636, Sept. 2011.

[4] I. Shen, Zhang, L. ANN-Based Algorithm for Destriping and Inpainting of Remotely Sensed Images. IEEE Trans. Geosci. Remote Sens. 2019, 47, 1492-1502.

[5] D. Bouali, Ladjal, S. Toward Optimal Destriping of MODIS Data Using a Unidirectional Variational Model. IEEE Trans. Geosci. Remote Sens. 2016, 49, 2924-2935.

[6] A. Zhou, Fang, H.; Yan, L.; Zhang, T.; Hu, J. Removal of stripe noise with spatially adaptive unidirectional total variation. Optik 2016, 125, 2756-2762.

[7] Shaker A. S., "DE-striping Augmented Images of Blood Cells using Deep Convolutional Neural Network," Journal of Al-Qadisiyah for Computer Science and Mathematics, vol. 13, no.2, pp:56-63, July 2021. https://doi.org/10.29304/jqcm.2021.13.2.8201

[8] P. Gong, R. Biging, G.S., Larrieu, M.R., "Estimation of deep learning models using vegetation Indices Derived from Hyperion Hyperspectral Data," IEEE Transactions on Geoscience and Remote Sensing vol. 2, no. 7, pp. 1- 6, 2015.

[9] M. Gross, V. Klemas, "The use of DCNN and Imaging Spectrometer data to differentiate marsh vegetation," Remote Sensing of Environment vol. 22, no. 4, pp. 60-66, 2018.

[10] N. Rinker, "Hyperspectral Imagery, A New DCNN Technique for Targeting and Intelligence," Presented at the Army Science Conference, Durham, vol. 6, no. 4, pp. 20-26, Sept. 2016. 
Asian Journal of Applied Sciences (ISSN: 2321 - 0893)

Volume 9 - Issue 4, August 2021

[11] G. Ghosh, S. Kumar, K. Saha, "Hyperspectral Satellite Data in Mapping Salt-Affected Soils Using Linear Spectral Unmixing Analysis," Journal of the Indian Society of Remote Sensing, vol. 8, no. 11, pp. 71- 76, 2016. 